\title{
Modelos Analógicos para la Visualización del Tiempo
}

\section{Analogical Models for Visualization of Time}

\author{
Rodrigo Martin Iglesias \\ Universidad de Buenos Aires, Argentina \\ rodrigo.martin.iglesias@gmail.com
}

\begin{abstract}
We present the foundations of a research project which is currently in development. This project aims to explore new dimensions and configurations of a traditionally one-dimensional Time. Initially we have systematized the material produced since 2005 with students of History of Architecture in relation to analogical (multidimensional) Timemaps, while we sought for theoretical tools that allows its interpretation. We have characterized different times and durations, from phenomenological aspects of temporal construction and perception, to historical and natural phenomena of long duration. Finally, we explored analogical and digital visualization tools to understand the intrinsic complexity of Time and its models.
\end{abstract}

Keywords: Visualization; Time; Timemaps; Analogical models; Topoheterochronics.

\section{Introducción}

Presentamos aquí las bases y resultados parciales de un proyecto de investigación que se encuentra en desarrollo actualmente. El referido proyecto pretende explorar nuevas dimensiones $\mathrm{y}$ configuraciones de un tiempo tradicionalmente unidimensional y lineal. En la etapa inicial se ha pretendido sistematizar el material producido durante más de seis años con los alumnos de Historia de la Arquitectura en relación a los Mapas de tiempo multidimensionales y encontrar o diseñar instrumentos teóricos para interpretarlos. A tal fin se ha propuesto, por un lado, la investigación y caracterización de los diferentes Tiempos y Duraciones, desde los aspectos fenoménicos de la percepción y construcción temporal, a los fenómenos históricos y naturales de larga duración. Por otro lado, se ha buscado encontrar y diseñar herramientas de representación y visualización que ayuden a la comprensión de los fenómenos anteriormente citados en su intrínseca complejidad. Asimismo, se encuadra el trabajo en una línea de exploración de nuevos instrumentos pedagógicos en la enseñanza dentro del marco de la didáctica constructivista, además de una intrínseca reflexión crítica sobre las herramientas tradicionales y sus conceptualizaciones, tema sobre el que ya hemos hablado en eventos científicos anteriores (Martin Iglesias, 2008, 2010). Cabe citar como ejemplo ilustrativo la problemática inicial que se plantea en el momento de enfrentarse con la práctica del taller: la evaluación y el adecuado uso de los conocimientos previos del alumno, momento crucial del proceso educativo, al que no se le presta la debida atención. Decimos crucial porque se sitúa como instancia anterior a la propia práctica desde el punto de vista del diagnóstico del estado en el que se encuentran los alumnos respecto de tales conocimientos. La conciencia en el uso de estos preconceptos y aprendizajes previos, por parte del docente, es lo que propicia que el aprendizaje sea una actividad significativa, en particular cuando se trata de la relación entre el conocimiento nuevo y el que el alumno ya posee como precondición de la comprensión.

\section{Topoheterocronías}

Se toma como punto de partida la trasgresión crítica de la clásica línea de tiempo, como representación gráfica de una secuencia de eventos, que obviamente contiene en sí una idea/concepto de cronología directamente relacionada con un paradigma cultural que nos lleva a asociar la antecedencia a la causalidad y que oculta una metafísica teleológica del tiempo histórico. Esto aparece evidentemente tanto en nuestras agendas o calendarios, como en los discursos más elaborados sobre fenómenos históricos y los relatos que generalmente son construidos a su alrededor. Al mismo tiempo, las representaciones tradicionales no incluyen todos los eventos, sino solamente aquellos que se consideran relevantes desde determinado punto de vista, en general en relación a los cambios o repercusiones que estos supuestamente generan a posteriori. Lo cual confirma la existencia de una lógica causalista y demuestra hasta que punto este tipo de construcciones son producto de una subjetividad cultural e ideológica que finalmente establece las conexiones de eventos y consecuencias de manera tautológica.

Más allá de que la existencia de esta concepción del tiempo también pertenece a la historia y puede realizarse una arqueología de su constitución como dispositivo cultural, también es interesante resaltar que al interior del paradigma se han producido múltiples exploraciones alternativas que van desde la representación de las digresiones narrativas como caminos no lineales en el Tristram Shandy de Laurence Sterne alrededor de 1760, pasando por las Ucronías contrafactuales de Charles Renouvier, a los argumentos de Henri Bergson a fines del siglo XIX 
por una distinción entre la concepción matemática y homogénea del tiempo y la experiencia heterogénea de la duración, la cual obviamente resulta imposible de representar en el modelo lineal.

\section{Temporalidades: Tiempos Hegemónicos y Alternativos}

"¿Qué es, entonces, el tiempo? Si nadie me lo pregunta, lo sé; si quiero explicárselo a quien me lo pregunta, no lo sé. Sin embargo, con toda seguridad afirmo saber que, si nada pasase, no habría tiempo pasado, y que si nada sobreviniese, no habría tiempo futuro, y que si nada hubiese, no habría tiempo presente". Esta frase, extraída de las Confesiones de San Agustín (2010), nos muestra de qué modo el tiempo es algo doble, intensamente ambiguo, algo que no podemos explicar, pero que sin embargo existe como una certeza para nuestra conciencia.

Existen múltiples representaciones mentales del tiempo, relacionadas profundamente con las percepciones que tenemos de él y con las conceptualizaciones que hacemos a partir de las mismas, estas representaciones están imbricadas con patrones culturales que configuramos y nos configuran. Desde que nacemos nuestras experiencias con el tiempo aparecen mediadas por una serie de convenciones sociales, pautas culturales y patrones de actuación, que tienen por función regular su uso y que el mismo sea común a un colectivo determinado. En palabras de Jeremy Rifkin: "Cada cultura posee su propio y único conjunto de huellas digitales temporales. Conocer a un pueblo equivale a conocer los valores del tiempo que han adoptado para vivir. Para conocernos a nosotros mismos, la razón por la que influimos unos sobre otros y sobre el mundo de la manera en que lo hacemos, debemos comprender en primer lugar la dinámica temporal que rige el tránsito humano en la historia" (Rifkin, 2004). Estas prácticas, dispositivos y procedimientos, que regulan culturalmente nuestras temporalidades, no son innatas, normales, ni consustanciales de la naturaleza humana como algunos quieren hacernos creer. Existen desde siempre toda una serie de conflictos y disputas de poder por imponer una visión cultural por sobre otras, un paradigma espacio temporal por sobre otros, luchas que establecen jerarquías, dominios, predominancia de algunos modos de sentir y pensar el tiempo. Como consecuencia de esto, existen tiempo "hegemónicos" y tiempos "contrahegemónicos" o alternativos, tiempos que se proponen como universales y tiempos que plantean modelos opuestos o simplemente diferentes para nuestras temporalidades. Dice Roger Caillois en Temps circulaire, temps rectiligne (1975): “...desde su nacimiento, uno está tan acostumbrado a la concepción del tiempo aceptada por quienes lo rodean, que no sería capaz de imaginar que existe otra que a otros les parezca tan natural y lógica como a él le parece la propia. No sospecha que haya aceptado inconscientemente sus implicaciones inexorables. Ignora que cada cultura posee una representación particular de la sucesión histórica y que su propia concepción del mundo, su universo moral, quizás incluso las normas prácticas de su conducta cotidiana aparecen insidiosamente modificadas en ella".

Nuestra concepción del tiempo, la concepción hegemónica en el occidente de raíz judeo-cristiana y greco-romana, es la de un tiempo uniforme, unívoco, universal, uno. Un tiempo, El Tiempo, que aparece fuertemente ligado a la cosmovisión griega clásica y sobre todo a las explicaciones del movimiento. Ya Aristóteles en su Física (1995) nos adelanta: "El tiempo es, pues, el mismo, ya que el número es igual y simultáneo para la alteración y el deslazamiento. $Y$ por esta razón, aunque los movimientos sean distintos y separados, el tiempo es en todas partes el mismo, porque el número de los movimientos iguales y simultáneos es en todas partes uno y el mismo". Un tiempo que es en todas partes (y para todos) el mismo, igual, simultaneo, y que no casualmente será tomado por la ciencia desde sus etapas formativas para imponer un paradigma por sobre otros, el único tiempo Verdadero. Un tiempo que tiene dirección, pero que es reversible, porque en teoría todos los fenómenos físicos son reversibles. En The Mathematical Principles of Natural Philosophy, Isaac Newton (1993) confirma nuestras apreciaciones: "El tiempo absoluto, verdadero y matemático, por sí mismo, y por su propia naturaleza fluye uniformemente, sin consideración por nada externo. De otro modo se nombra la duración: el tiempo relativo, aparente y corriente, es una medida de la duración sensible y externa (ya sea exacta o irregular) por medio del movimiento, la cual es corrientemente usada en lugar del tiempo verdadero". Es muy interesante notar que en esta cita ya aparece otro tiempo, la duración, el tiempo Bergsoniano, pero asoma denostado frente a un tiempo "verdadero", es ese otro tiempo "aparente" y "corriente", es aquel fundado en la percepción, en la experiencia, el del ciudadano común, que claramente no sirve a la ciencia por ser subjetivo, fundamentalmente cuando se persigue lo "absoluto".

Luego la filosofía se encargará de dudar, de repensar, de poner en crisis al paradigma, que vale la pena aclarar, sigue regulando nuestro comportamiento y nuestras prácticas sociales, a pesar de que la propia ciencia ya se ha ocupado de demostrar que no es absoluto ni verdadero. "El tiempo que trato de determinar es siempre 'tiempo para', tiempo para hacer esto o aquello, el tiempo que puedo permitirme para, el tiempo que me puedo tomar para realizar esto o aquello, el tiempo que me tengo que tomar para llevar a término esto o aquello. El mirar-el-reloj se funda en un tomarse-tiempo y surge de él. Para poder tomarme tiempo, tengo que tenerlo en alguna parte", esta cita de Los problemas fundamentales de la fenomenología, de Martin Heidegger (2000), nos coloca frente a una visión muy relevante del tiempo de la modernidad, un tiempo que se tiene, que se posee y que por lo tanto, se puede vender o alquilar, un tiempo del reloj de la fábrica, de la productividad de la industria, un bien del mercado capitalista. Simultáneamente, se sitúa de manera intrínseca como un tiempo de uso, un tiempo en función de algo, funcional, "tiempo para" en palabras de Heidegger. Una perspectiva que 
para los arquitectos es evidentemente coherente con la misma concepción aplicada al espacio y a las formas, que pretende regular los modos de habitar, predeterminarlos, uniformarlos, y de este modo fugar hacia una utopía del bien común, que paradójicamente confunde el bien con los bienes. Por otro lado, los empiristas hacen vacilar la idea del tiempo absoluto, exterior a nosotros mismos, y se fijará en las sucesiones, las series, los procesos, los ritmos, las continuidades y las discontinuidades. Un tiempo más humano, pero también más relativo, que aparece a partir de la observación y que vuelve a los orígenes del nacimiento del paradigma en el movimiento y el cambio, podríamos decir que rescata el legado de Heráclito frente al triunfo de los seguidores de Parménides: "Siempre que no tenemos percepciones sucesivas, no poseemos la noción del tiempo, aunque exista una sucesión real en los objetos. De este fenómeno, lo mismo que de muchos otros, podemos concluir que el tiempo no puede hacer su aparición en el espíritu solo o acompañado de un objeto fijo e inmutable, sino que se descubre siempre por alguna sucesión perceptible de objetos mudables" (Hume, 2002). Sería interesante pensar que consecuencias podría haber tenido pensar la arquitectura desde esta noción que opone la aparición de la experiencia de lo temporal a los objetos fijos e inmutables. A una arquitectura que incluso hoy en día se concibe como objetual y terminada, metáfora material de la trascendencia.

Una de las discusiones más sugestivas es aquella que, a partir de nuestra experiencia de la sucesión, de lo que ya no es y de lo que todavía no fue, plantea las diferentes versiones de la subdivisión del transcurrir en lo pasado, lo presente y lo futuro. En su Lógica del sentido (1989), Gilles Deleuze nos presenta inicialmente un presente continuo, aunque luego se ocupará de relativizar esta concepción, e incluso contradecirla: "únicamente el pasado y el futuro insisten o subsisten en el tiempo. En lugar de un presente que reabsorbe el pasado y el futuro, un futuro $y$ un pasado que dividen el presente en cada instante, que lo subdividen hasta el infinito en pasado y futuro, en los dos sentidos a la vez". Un presente infinitesimal frente a un presente eterno. Una discusión filosófica que se hunde en la noche de los tiempos. Quizás como anverso de esta dialéctica aparecen esos otros tiempos, esos tiempos alternativos de otras culturas, uno de los cuales sin duda nos enriquece de sólo pensarlo, el tiempo de la cultura china, una forma de concebir la temporalidad más compleja y dinámica, un tiempo tejido al espacio y al evento, una serie de eventualidades imbricadas en momentos y lugares: "el tiempo chino es un tiempo propio, interior a las cosas, o mejor, a los procesos y a las situaciones. Más que tiempo, hay tiempos. Tan entreverado está el tiempo con el acontecimiento que no sólo es más bien el tiempo del acontecimiento (un tiempo creado por ese concreto acontecer) sino que se anuda también con el espacio; un espacio que, igualmente, tampoco es el espacio sino su espacio, el lugar que el propio acontecer determina y carga con sus propiedades" (Lizcano, 1992). $Y$ es significativo trazar aquí la diferencia que antes mencionábamos, estos otros tiempos son distintos, simplemente inconmensurables, no se pueden comparar con los nuestros, no son contrahegemónicos, no vienen a ponen en crisis nada, ni a oponerse a nada, son otros tiempos.

Por último, el tiempo histórico, ese que nos atrae particularmente por nuestras experiencias docentes. La historia es en si misma una forma de temporalidad, una forma conectada con los tiempos absolutos o relativos, universales o humanos, de los que hablamos anteriormente. Por ejemplo, en el siguiente texto de Benjamin vemos aparecer de nuevo esta dialéctica del tiempo absoluto de la ciencia, del presente infinitesimal, frente al presente continuo de la simultaneidad: "La historia es objeto de una construcción cuyo lugar no está constituido por el tiempo homogéneo y vacío, sino por un tiempo pleno, 'tiempo-ahora'. Así la antigua Roma fue para Robespierre un pasado cargado de 'tiempo-ahora' que él hacía saltar del continuum de la historia" (Benjamin, 1982). Sin embargo, el punto fundamental de esta cita es la idea de la historia como construcción, es lo que da sentido a la continuidad, todo pasa simultáneamente en la mente del historiador, o dicho de otro modo, todo existe al mismo tiempo. No obstante, la condición narrativa de la historia tal y como la conocemos va acompañada de una temporalidad lineal, de lectura, de relato, que obviamente admite complejidades, ramificaciones, bucles y paralelismos, pero que como dice Paul Ricoeur en El tiempo relatado (1992): "es correlativo del tiempo implicado en la narración de los hechos. Relatar, en efecto, toma tiempo, y sobre todo organiza el tiempo. El relato es un acto configurante que, de una simple sucesión, obtiene formas temporales organizadas en totalidades cerradas. Ese tiempo configurado está estructurado en tramas que combinan intenciones, causas y azares". De todos modos, aquí llegamos a un punto donde simplemente se abren nuevas discusiones y polémicas, el rol de las intenciones en la historia, así como el concepto de causalidad, son sólo algunas de las cuestiones pendientes, por no hablar del problema del relato en sí y de las características estructurales que traslada la narración a la construcción histórica.

\section{Visualizaciones: Representación y Cognición}

Podemos decir que todas las anteriores reflexiones sobre el tiempo tienen sentido para nuestro trabajo en función de un objetivo, establecer alternativas al relato historicista a partir de modelos espaciales alternativos. Las representaciones de los acontecimientos, sus relaciones lógicas y topológicas, nos permiten investigar sobre nuevos instrumentos cognitivos para pensar la historia. Las representaciones gráficas y espaciales del tiempo abren nuevas perspectivas sobre la temporalidad a través de analogías y metáforas visuales. La manera en la cual nuestra mente construye nociones de tiempo a través de analogías y la importancia que esto tiene en el resto de nuestro pensamiento ya aparece con claridad en la Crítica de la razón pura (1978) de Immanuel Kant: "el tiempo no puede ser una determinación de fenómenos externos; ni pertenece a una figura ni a una posición, etc., y en cambio, determina la relación de las representaciones en nuestro estado interno. $Y$, precisamente, porque esa intuición interna no da figura alguna, tratamos de suplir este defecto por 
medio de analogías y representamos la sucesión del tiempo por una línea que va al infinito, en la cual lo múltiple constituye una serie, que es sólo de una dimensión; y de las propiedades de esa línea concluimos las propiedades todas del tiempo, con excepción de una sola, que es que las partes de aquella línea son a la vez, mientras que las del tiempo van siempre una después de la otra". Aquí observando las restricciones que impone la analogía espacial del tiempo lineal, pero que evidentemente resulta trasladable a cualquier representación mental o corporal del tiempo. Una cuestión sobre la cual la psicología también ha trabajado desde sus inicios: "Nuestra representación abstracta del tiempo parece más bien estar enteramente tomada del modo de trabajo del sistema $P$ Cc [Percepción-Conciencia], y corresponder a una autopercepción de éste" (Freud, 1997). Estamos convencidos de que estas restricciones intrínsecas a todo modelo, a toda metáfora, a toda representación, no deben impedir aprovechar la riqueza que nos ofrecen como herramientas del pensamiento, suerte de asistentes cognitivos, y de la potencia que tienen en la investigación, la enseñanza y el aprendizaje, frente a lo establecido, institucionalizado o hegemónico.

Muestras de estos modelos alternativos hay muchas (cfr. Rosenberg, 2010), desde las líneas genealógicas (Línea genealógica de Maximiliano I, Albrecht Dürer, 1516) o las representaciones de la historia (Historia universal, Johannes Bruno, 1672), hasta las exploraciones artísticas de Ward Shelley (Who Invented the Avant Garde? o Frank Zappa), pasando por los modelos híbridos, radiales (Spiegazione della Carta Istorica dell'Italia, Girolamo Andrea Martignoni, 1721), cíclicos (La rueda de la moda, J J Grandville, 1844), de flujos (Strom der Zeiten, Friedrich Strass, 1849 o The Histomap, John Sparks, 1931), o incluso variaciones de las representaciones lineales como la famosa Línea de tiempo de Dubourg. De cualquier manera, estos ejemplos sólo los utilizamos como antecedentes en la investigación o disparadores en el aprendizaje, dentro de una propuesta mayor que apunta a las exploraciones individuales de posibles cartografías del tiempo y la historia que fomenten nuevas construcciones narrativas o incluso nuevos tipos de historicidad.

"Y el fin de toda exploración será llegar a donde empezamos / Y conocer el lugar por primera vez..." T. S. Eliot, Cuatro cuartetos.

\section{Agradecimientos}

Al subsidio UBACYT 20020100100784 y a la beca doctoral, otorgados por la Universidad de Buenos Aires.

\section{Referencias}

Aristóteles (1995) Física. Trad. de Guillermo R. de Echandía. Madrid: Gredos.

Benjamin, W. (1982), "Tesis de filosofía de la historia." En: Discursos interrumpidos. Madrid: Taurus.

Callois, R. (1975) Obliques. Précédé de Images, images. Paris: Stock.

Deleuze, G. (1989) Lógica del sentido. Traducción. Miguel Morey y Víctor Molina. Barcelona: Paidós.

Freud, S. (1997) Obras Completas, Tomo XVIII: Más Allá del principio de placer, Psicología de las masas y Análisis del yo y otras obras. Buenos Aires: Amorrortu.

Heidegger, M. (2000) Los problemas fundamentales de la fenomenología. Madrid: Trotta. Trad. y prólogo de Juan José García Norro. Título original: Die Grundprobleme der Phänomenologie, V. Klostermann, Frankfurt a. M., 1975. Edición de F.-W. von Herrmann.

Hume, D. (2002) Tratado de la naturaleza humana. Estudio preliminar, traducción y notas, Félix Duque. Traducción de: A treatise of human nature. Madrid: RBA.

Kant, I. (1978) Crítica de la razón pura. Título original: Kritik der reinen Vernunft. Prólogo, traducción, notas e índices: Pedro Ribas. Madrid: Alfaguara.

Lizcano, E. (1992) "El tiempo en el imaginario social chino", Archipiélago, no 10/11, pp. 59-67.

Martin Iglesias, R. A. (2008). Hacia una arquitectura de la historia, un enfoque constructivista. Actas del III Encuentro Taller de Docentes de Historia de la Arquitectura, el Diseño y la Ciudad; Buenos Aires. Facultad de Arquitectura, Diseño y Urbanismo, Universidad de Buenos Aires.

Martin Iglesias, R. A. (2010).La enseñanza de la historia frente a la amnesia proyectual. Actas del IV Encuentro-Talleres de Docentes e investigadores en Historia del Diseño, Arquitectura y Ciudad. Montevideo. Facultad de Arquitectura, Universidad de la República.

Newton, I. (1993) Principios matemáticos de la Filosofía natural [Philosophiae Naturalis Principia Mathematica]. Estudio preliminar y traducción Antonio Escohotado. Barcelona: Altaya.

Ricoeur, P. (1992) La función narrativa y el tiempo, Buenos Aires: Almagesto.

Rifkin, J. (2004) Las Guerras del Tiempo, El Siglo de la Biotecnología y El sueño europeo. Barcelona: Paidós.

Rosenberg, D. \& Grafton, A. (2010) Cartographies of Time: a history of the timeline. Princeton, NJ: Princeton Architectural Press.

San Agustín (2010). Confesiones. Madrid: Gredos. 